\title{
Citrullus lanatus: un nuevo hospedante natural del Groundnut ringspot orthotospovirus en Argentina
}

\author{
Pozzi, E. A., Luciani, C. E., Giovani Celli, M., Conci, V. C. y Perotto, M. C.
}

DOI: 10.31047/1668.298x.v38.n1.26732

\begin{abstract}
RESUMEN
Los orthotospovirus causan graves daños económicos, siendo una limitación importante para la producción y la calidad en distintos cultivos. Los objetivos de este trabajo fueron identificar el agente causal de los síntomas observados en sandía en lotes de producción de Santiago de Estero y analizar su filogenia. Se realizaron muestreos en lotes de producción de sandía (Citrullus lanatus) durante la campaña 2017/2018 en la provincia de Santiago del Estero. Los resultados de este trabajo mostraron en las muestras de sandía la presencia de viriones característicos del género Orthotospovirus, además fueron positivas a pruebas serológicas y moleculares con reactivos específicos (DAS-ELISA y RTPCR) confirmando la presencia del Groundnut ringspot orthotospovirus (GRSV). Los fragmentos genómicos fueron amplificados, secuenciados y depositados en GenBank, acceso No MK680832 y MK680833. El análisis filogenético de las secuencias parciales de nucleótidos de la proteína $\mathrm{N}$ mostró que los aislamientos de GRSV provenientes de Argentina (hospedante sandía) fueron agrupados con alto valor de confianza. Estas secuencias se ubicaron en un clúster formado por secuencias del continente americano que se separaron de las de origen africano. Este trabajo constituye el primer reporte de la presencia del GRSV en cultivo de sandía (Citrullus lanatus) en Argentina.
\end{abstract}

Palabras claves: GRSV; sandía; RT-PCR; ELISA.

Pozzi, E. A., Luciani, C. E., Giovani Celli, M., Conci, V. C. and Perotto, M. C. 2020. Citrullus lanatus: a new natural host of Groundnut ringspot orthotospovirus in Argentina. Agriscientia 38: 71-78

\section{SUMMARY}

Orthotospoviruses cause serious economic damage, being a major limitation for production and quality in different crops. The objectives of this work were to detect the presence of orthotospovirus that are infecting cucurbit crops in the country and analyze its phylogeny. Samples were taken in watermelon (Citrullus lanatus) production lots during 2017/2018 in Santiago del Estero province. The 
results of this work showed in the watermelon samples showed the presence of characteristic virions of the Orthotospovirus genus, and were positive to serological and molecular tests with specific reagents (DAS-ELISA and RT$\mathrm{PCR}$ ) confirming the presence of groundnut ringspot orthotospovirus (GRSV). Genomic fragments were amplified, sequenced and deposited in GenBank, access $n^{\circ}$ MK680832 and MK680833. Phylogenetic analysis of N-protein nucleotide partial sequences showed that GRSV isolates from Argentina (watermelon host) were grouped into a new group with high bootstrap value. These sequences were placed in a cluster formed by sequences from the American continent that were separated from those that conformed the group of sequences of African origin. This work is the first report of the presence of the GRSV in watermelon (Citrullus lanatus) cultivation in Argentina.

Key words: GRSV; watermelon; RT-PCR; ELISA.

Pozzi, E. A. (ORCID: 0000-0002-2768-3142): Universidad Nacional de Córdoba, Facultad de Ciencias Agropecuarias. Consejo Nacional de Investigaciones Científicas y Técnicas (CONICET), Córdoba, Argentina. Unidad de Fitopatología y Modelización Agrícola (UFyMA). Giovani Celli, M. (ORCID:0000-0002-9256-6305): Consejo Nacional de Investigaciones Científicas y Técnicas (CONICET), Córdoba, Argentina. Unidad de Fitopatología y Modelización Agrícola (UFyMA). Luciani, C. E. (ORCID: 0000-0001-6817-0549), Conci, V. C. (ORCID: 0000-0002-1688-9567) y Perotto, M. C. (ORCID 00000002-3408-0067): Consejo Nacional de Investigaciones Científicas y Técnicas (CONICET), Córdoba, Argentina. Unidad de Fitopatología y Modelización Agrícola (UFyMA). Instituto Nacional de Tecnología Agropecuaria (INTA), Centro de Investigaciones Agropecuarias (CIAP), Instituto de Patología Vegetal (IPAVE). Correspondencia a: perotto.cecilia@inta.gob.ar.

\section{INTRODUCCIÓN}

Las enfermedades provocadas por orthotospovirus causan importantes daños en diversos cultivos en todo el mundo generando graves pérdidas económicas (Goldbach y Kuo, 1996; Mumford, Barker y Wood, 1996; Pappu, Jones y Jain, 2009).

Estos virus infectan principalmente cultivos ornamentales y hortícolas, siendo Orthotospovirus el único género de la familia Tospoviridae (Maes et al., 2019) cuyos miembros infectan plantas, mientras que los integrantes de los demás géneros de esta familia infectan invertebrados (Holkar, Mandal, Reddy y Jain, 2019). Se transmiten exclusivamente por trips (Thysanoptera: Thripidae) de manera persistente y propagativa (Jones, 2005), por lo que el virus se replica en el insecto, circula a través del cuerpo y persiste a través de las diferentes etapas de desarrollo. La adquisición del virus depende de la etapa de desarrollo del vector; solo los trips que adquieren el virus en el primer estadio larvario son capaces de transmitirlo y a medida que se desarrollan, la eficiencia de la adquisición disminuye (Rotenberg, Jacobson, Schneweis y Whitfield, 2015). De las 1710 especies conocidas de Thripidae, se conocen hasta la actualidad solo 14 especies que son capaces de transmitir orthotospovirus (Riley, Joseph, Srinivasan y Diffie, 2011).

El genoma de los orthotospovirus tiene una organización conservada compuesta por tres segmentos clasificados como pequeño (S), medio (M) y grande (L). El segmento S codifica la proteína de la nucleocápside (N) y la proteína no estructural (NS) (Turina, Tavella y Ciuffo, 2012). Tanto la proteína $\mathrm{N}$ como la NSs, juegan un importante rol en la infección viral, la patogénesis y el ensamblaje de la partícula viral (Olaya, Adhikari, Raikhy, Cheng y Pappu, 2019).

Las distintas especies de Orthotospovirus se diferencian por la especificidad del vector, su rango de hospedante, las relaciones serológicas de la proteína $\mathrm{N}$ y la secuencia genómica del gen que codifica esta proteína, que debe mostrar menos del $90 \%$ de identidad con la de cualquier otra especie descrita para este género según el International Committee on Taxonomy of Viruses (ICTV) (Plyusnin et al., 2011).

En el mundo se han reportado varias especies del género Orthotospovirus que infectan cucurbitáceas: watermelon silver mottle 
orthotopovirus (WSMoV) (Rao, Liu, Wu y Li, 2011), tomato spotted wilt orthotospovirus (TSWV) (Yeh et al.,1992), zucchini lethal chlorosis orthotospovirus (ZLCV) (Bezerra et al., 1999), watermelon bud necrosis orthotospovirus (WBNV) (Jain, Pappu, Pappu, Reddy y Vani, 1998), melon yellow spot orthotospovirus (MYSV) (Kato, Handa y KameyaIwaki, 2000), melon severe mosaic orthotospovirus (MeSMV) (Ciuffo et al., 2009) y groundnut ringspot orthotospovirus (GRSV) (Spadotti, Leão, Rocha, Pavan y Krause-Sakate, 2014).

EL GRSV ha sido detectado en Brasil, recientemente reportado en infecciones naturales en sandía y pepino (Spadotti et al., 2014; Leão et al., 2015). En Argentina este virus ha sido reportado en pimiento, tomate, tabaco, maní (Dewey, Semorile y Grau, 1996; de Breuil et al., 2007). Este patógeno ha mostrado una amplia distribución geográfica y extenso rango de hospedantes (Lecoq y Katis, 2014).

Como vectores de GRSV han sido citados Frankliniella schultzei, que transmite muy eficientemente el virus, y F. occidentalis, con menor eficiencia (Wijkamp, Almarza, Goldbach y Peters, 1995; Nagata, Almeida, Resende y De Ávila, 2004). Estos insectos han sido reportados en Argentina en diversos cultivos (SINAVIMO, $2019 \mathrm{a}, \mathrm{b}$ ).

Se encontró que los orthotospovirus estaban vinculados en clados al grupo de origen geográfico en función de la similitud de secuencia genómicas y tienden a compartir distribuciones geográficas y vectores (Oliver y Whitfield, 2016).

En Argentina se han detectado potyvirus y cucumovirus en cucurbitáceas (Nome, March y Giorda, 1974; Gracia y Feldman, 1986; Perotto, Celli, Pozzi, Luciani y Conci, 2016; Perotto et al., 2017) y se ha hecho reporte de la presencia de un orthotospovirus (el ZLCV) en zapallos (Pozzi, Luciani, Celli, Conci y Perotto, 2019). Los objetivos de este trabajo fueron identificar el agente causante de los síntomas observados en sandía en lotes de producción de Santiago de Estero y analizar su filogenia.

\section{MATERIALES Y MÉTODOS}

Se realizaron muestreos durante la campaña 2017/2018 en la provincia de Santiago del Estero y se recolectaron muestras (hojas) de un total de 90 plantas de sandía (Citrullus lanatus). El material presentaba síntomas de deformaciones, reducción de la lámina foliar, manchas amarillas y puntos necróticos. Esta llamativa sintomatología de daños necróticos en el tejido foliar ya mencionados para orthotospovirus en cucúrbitas (Leão et al., 2015) nos daba indicios de la presunta presencia de algún virus que se encuentre dentro de este género (Figura 1).

La observación de partículas virales se efectuó en cortes ultrafinos de tejido infectado; para ello se cortaron pequeños trozos de hojas con síntomas y se fijaron en paraformaldehido/ glutaldehido seguido de tetróxido de osmio $1 \%$. Para la deshidratación del tejido se empleó serie de acetona (10-100 \%) y se incluyó en resina de baja viscosidad. Se realizaron cortes con ultramicrotomo y se contrastó con citrato de plomo pH12 en caja de Petri con perlas de hidróxido de sodio para evitar la precipitación de sales y luego con acetato de uranilo $2 \%$. Las observaciones se hicieron en microscopio electrónico de transmisión JOEL 1220 EXII.

Se realizó una prueba inmuno enzimática de doble sándwich de anticuerpo (DAS-ELISA), según Clark y Adams (1977), utilizando antisuero de amplio espectro para la detección de orthotospovirus (BIOREBA SRL Latin América, Mar del Plata, Argentina), siguiendo la metodología

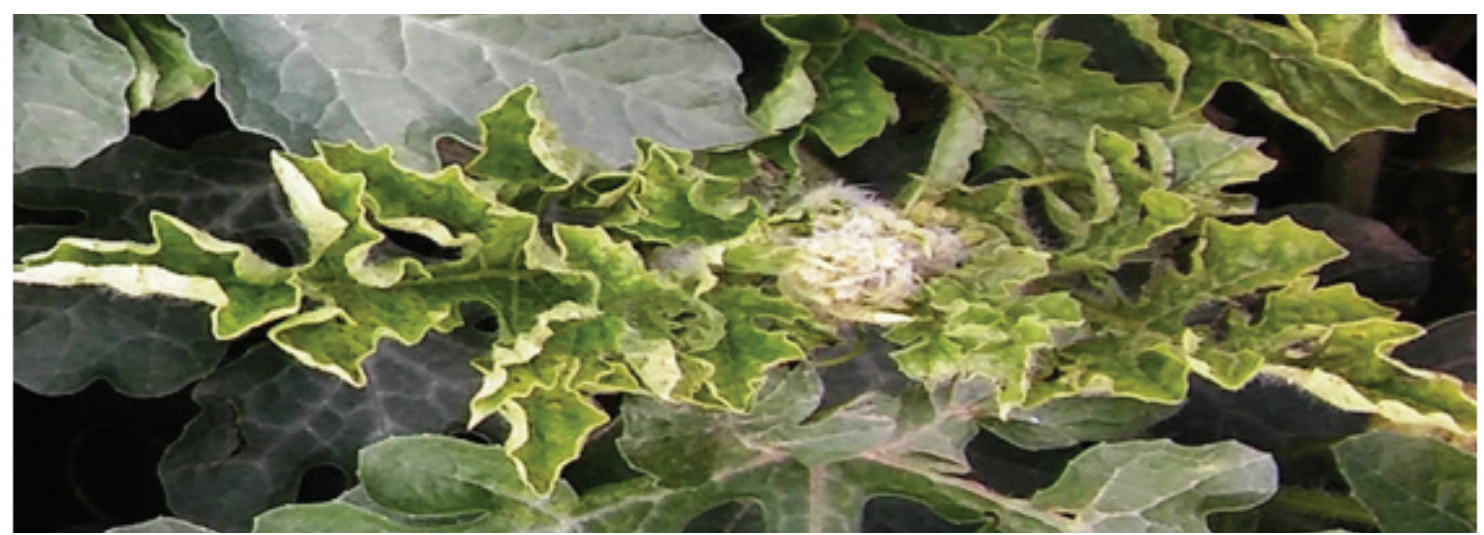

Figura 1. Lesiones necróticas y mosaico en las hojas de sandía en el campo causadas por groundnut ringspot orthotospovirus (GRSV) 
descripta por el fabricante. Las especies de este género se han clasificado en serogrupos utilizando anticuerpos contra la proteína nucleocápside (N). Este reactivo reconoce los serogrupos I, II y III dentro del género Orthotospovirus.

La detección específica se realizó mediante la reacción en cadena de la polimerasa previa transcripción inversa (RT-PCR). El ARN total fue extraído de hojas de plantas con síntomas mediante el protocolo de Gambino, Perrone y Gribaudo (2008) modificado. Las modificaciones realizadas fueron: en la primera extracción, la mezcla cloroformo:alcohol isoamílico se agitó vigorosamente durante $10 \mathrm{~min}$ hasta obtener una emulsión. En la segunda extracción con cloroformo:alcohol isoamílico se mezcló la muestra durante 5 min vigorosamente hasta obtener una emulsión y luego del agregado de CILi se incubó la muestra a $-20^{\circ} \mathrm{C}$. Se usaron cebadores específicos para GRSV reportados por Boari et al. (2002). Se purificaron los productos de PCR de dos muestras con el kit Zymo (DNA clean \& concentrator, Zymo Research Corp., Irvine, California, Estados Unidos) y fueron enviados para secuenciar a la Unidad de Genómica del Instituto de Biotecnología - INTA (Argentina). Las alineaciones de secuencia se realizaron utilizando Clustal W. Todos los análisis moleculares se hicieron utilizando el programa MEGA versión 7 (Kumar, Stecher y Tamura, 2016) usando el método de distancias estadística Neighbor-Joining nearest-neighbor con el modelo Tamura-Nei con G (distribución gamma, 5 categorías) y 1000 réplicas para los análisis filogenéticos. Las secuencias obtenidas fueron comparadas con 20 secuencias parciales de nucleótidos de la proteína N de GRSV publicadas en GenBank (National Center for Biotechnology Information, Bethesda, MD, USA), utilizando BLAST (Basic Local Alignment Search Tool).

\section{RESULTADOS Y DISCUSIÓN}

Los orthotospovirus causan graves daños económicos a una amplia gama de cultivos en muchas partes del mundo (Bag, Mitter, Eid y Pappu, 2012), siendo una limitación importante para la productividad y calidad (Yin et al., 2014). Síntomas como mosaicos y lesiones necróticas en las hojas, malformaciones foliares y manchas necróticas en los frutos hicieron sospechar que el cultivo de sandía ( $C$. lanatus) podía estar infectado con GRSV, como ya fuera observado (Leão et al., 2015) y esto es una limitación para la calidad y la comercialización del producto.

Los resultados de este trabajo mostraron que siete muestras de sandía dieron resultados positivos a la prueba de DAS-ELISA con antisuero específico para Orthotospovirus (serogrupos I, II y III). Las especies de este género se han clasificado en serogrupos basados en relaciones serológicas, utilizando anticuerpos contra la proteína nucleocápside (N), y la homología de secuencia. En particular el GRSV, pertenece al serogrupo II de los Orthotospovirus (de Ávila et al., 1993). Además, la presencia de viriones de forma esférica de 80-120 nm de diámetro característicos de este género fueron observados al microscopio electrónico en las plantas infectadas (resultado no mostrado), coincidiendo con lo reportado por Plyusnin et al (2011).

La prueba de RT-PCR realizadas a las siete muestras que habían dado resultado positivo en las pruebas serológicas, confirmó la presencia

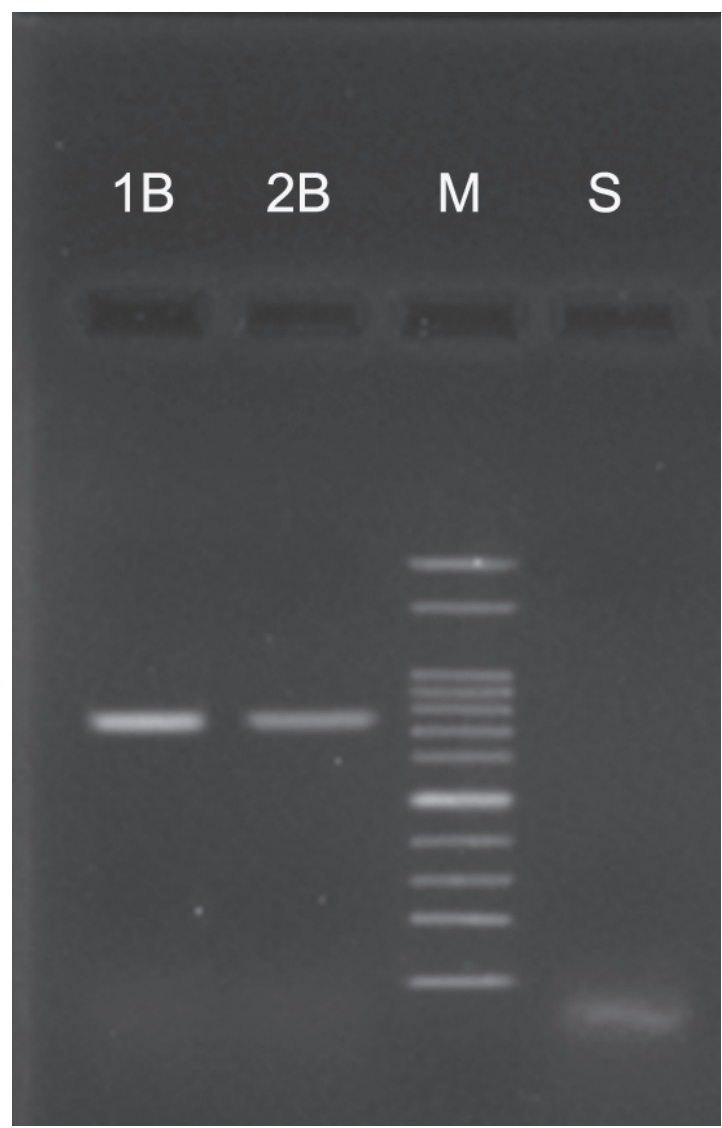

Figura 2. Corrida de electroforesis de producto de RT-PCR con cebadores específicos en gel de agarosa $2 \%$ para groundnut ringspot orthotospovirus (GRSV). (S) Planta de cucurbita utilizada como control sano; (M) marcador de peso molecular de $100 \mathrm{pb}$ (Ladder 100 pb precision, Embiotec S.R.L, Argentina); (1B y 2B) Plantas de C. lanatus (sandía) infectadas con groundnut ringspot orthotospovirus, mostrando amplificación de fragmento de $780 \mathrm{pb}$ 
del GRSV ya que se logró amplificar un fragmento del tamaño esperado (780 pb) con los iniciadores específicos para la detección de este virus (Figura 2).

Se lograron secuenciar los productos de RTPCR y se obtuvieron las secuencias genómicas provenientes de un fragmento del gen $\mathrm{N}$ de dos aislamientos virales. Estas secuencias fueron publicadas en GenBank con número de acceso MK680832 y MK680833.

Las secuencias de nucleótidos y aminoácidos para el aislamiento MK680832, en comparación con otros aislamientos de GRSV depositados en GenBank, revelaron que comparten el 97,62 \% de identidad de nucleótidos con un aislamiento de Brasil (MG797643) y el 98,74 \% de identidad de aminoácidos con un aislamiento de Estados Unidos (YP004421857.1). El segundo aislamiento obtenido, cuyo número de acceso fue MK680833, reveló identidades de secuencia de nucleótidos de $97,36 \%$ y de aminoácidos de 98,33\%, con el mismo aislamiento mencionado para MK680832. Las restantes relaciones de identidades para nucleótidos y aminoácidos no se muestran.

El análisis filogenético de las secuencias parciales de nucleótidos de la proteína $N$ mostró que los dos aislamientos de GRSV provenientes de muestras de sandía de Argentina fueron agrupados con un máximo nivel de confianza (Figura 3). Estas

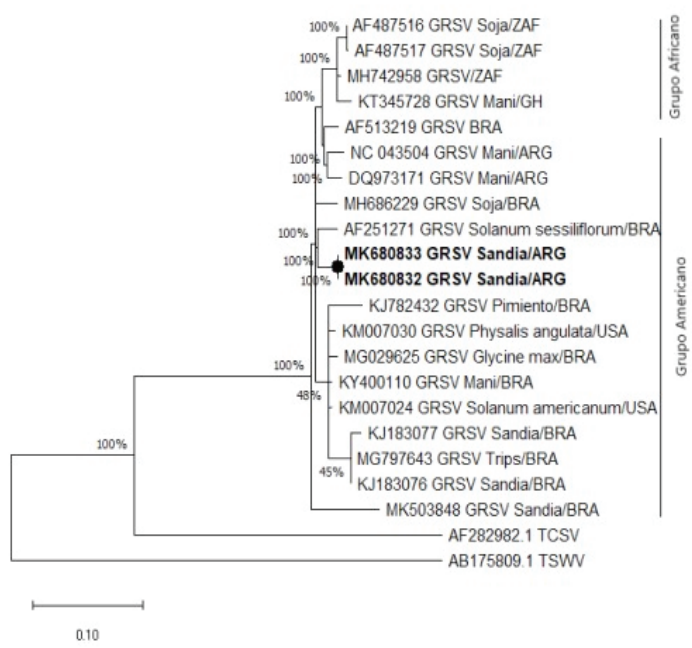

Figura 3. Árbol filogenético Maximun Likelihood basado en modelo Tamura-Nei construido con las secuencias parciales de nucleótidos de la proteína $\mathrm{N}$ de groundnut ringspot orthotospovirus (GRSV). En el árbol se observan los aislamientos de sandía argentinos (resaltados), secuencias de otros GRSV reportados (ZAF: Sudáfrica; GH: Ghana; USA: Estados Unidos; BRA: Brasil y ARG: Argentina), hospedantes de donde fueron aislados (cuando se cuenta con el mismo) y $N^{\circ}$ de acceso (1000 bootstrap) secuencias se ubicaron dentro del clúster formado por la mayoría de las secuencias provenientes del continente americano, separándose del que incluye las secuencias de origen africano. Se ha demostrado que los agrupamientos de otras especies de orthotospovirus no está afectados por el paso del tiempo sino que las poblaciones están agrupadas geográficamente (Tsompana, Abad, Purugganan y Moyer, 2005). Otros trabajos reportan que la dispersión geográfica del virus está relacionada con el hospedante que infecta y la distribución de vectores (Oliver y Whitfield, 2016), esto no coincide con los resultados de análisis filogenéticos obtenidos en este trabajo, en el cual los aislamientos de soja y maní están en ambos grupos geográficos, y nuestros aislamientos de cucurbitáceas comparten grupo con una solanácea, mientras que los de maní de Argentina se agruparon con el aislamiento brasileño de tabaco (AF513219). Cabe mencionar que los aislamientos reportados en este estudio se encuentran juntos y formaron un grupo con $100 \%$ de confiabilidad.

El hallazgo de GRSV en plantas de sandía podría indicar que este patógeno está aumentando el número de huéspedes infectados en nuestro país. La presencia de reservorios que actúen como hospedantes, para posibilitar que el patógeno sobreviva cuando el cultivo no está en producción, y un insecto vector, que se alimente tanto del reservorio como del cultivo, serían factores que permitirían la expansión del virus en las producciones anuales (Webster et al., 2015).

La presencia de trips fue detectada en el campo durante el muestreo en flores de sandía, si bien no se ha llevado a cabo la identificación de estas especies, se sabe que ambas especies de trips (F. schultzei y F. occidentalis) se encuentran frecuentemente alimentándose de las partes florales y $F$. schultzei es una especie polifágica (Milne, Khumlekhasing y Walter, 1996) por lo que podría ser responsable de la propagación de GRSV.

En Argentina el virus fue reportado en numerosas hortalizas y también en cultivos extensivos (SINAVIMO, 2019 c). En este trabajo se demuestra su presencia en los cultivos de sandía en el país, confirmando la importancia de este virus como patógeno muy polífago que puede causar importantes pérdidas del rendimiento en diversos cultivos. En Brasil el virus fue detectado en diferentes especies de cucurbitáceas (Spadotti et al., 2014; Leão et al., 2015) lo cual permite suponer que es probable que otras especies de esta familia, cultivadas en Argentina, estén igualmente afectadas por este patógeno. Esta enfermedad podría ser una potencial amenaza 
para otros cultivos y nuevas áreas geográficas. Además, se considera que la aparición de las enfermedades causadas por orthotospovirus es un proceso multifactorial que incluye virus, vectores, huéspedes, medio ambiente y comportamiento humano (Yin et al., 2014).

El manejo de estas enfermedades podría considerarse fácil debido a que se transmiten de manera persistente y un adecuado control químico del vector limitaría su dispersión, pero la alta capacidad para reproducirse en las zonas agrícolas, hace que las aplicaciones no sean altamente efectivas (Michelotto et al., 2019). Además, los trips pueden adquirir orthotospovirus sólo durante los estadios larvarios, mientras que tanto las larvas en estadios más avanzados, como los adultos, son capaces de transmitirlos (Loebenstein y Lecoq, 2012). Por otro lado, se ha reportado que algunos trips disminuyen la capacidad para transmitir los virus durante el desarrollo de adultos (German, Ullman y Moyer, 1992).

Estos conocimientos son de suma utilidad, para analizar el sistema agrícola en su conjunto y tomar decisiones acertadas al momento de su manejo. Prácticas como la utilización de agroquímicos para el control total y semanal de vectores trae aparejado dos consecuencias importantes para tener en cuenta en el momento del manejo de la enfermedad: por un lado los excesivos gastos que se generan al realizar demasiadas aplicaciones, y por otro, es posible que los vectores (independientemente de la especie de que se trate) se tornen resistentes/tolerantes a los productos químicos utilizados, siendo esto una amenaza potencial para futuros controles de las virosis transmitidos por ellos. Se necesitan nuevos enfoques que integren los procesos biológicos y ecológicos en la producción de alimentos, minimicen el uso de aquellos insumos que causan daños al medio ambiente, salud de los agricultores y los consumidores (Pretty, 2008).

\section{CONCLUSIONES}

Este es el primer reporte de la presencia de GRSV en una cucurbitácea en Argentina. El virus fue detectado en plantas de sandía y a partir de su detección, el análisis de este virus fue incorporado como rutina para el cultivo.

Los primeros orthotospovirus que han sido detectados infectando cultivos de cucurbitáceas en nuestro país son el GRSV y el ZLCV. El primero ha sido reportado en numerosas especies y países, mientras que el segundo sólo fue identificado en Brasil y en Argentina.
La identificación del GRSV en un nuevo hospedante (sandía) en nuestro país puede impactar sobre las estrategias de manejo en los cultivos hortícolas, debido a que este cultivo también podría estar actuando como un reservorio para la diseminación de la enfermedad. Por ello se considera de gran importancia continuar con estudios de prospección con el objeto de constatar si el virus está afectando otras de las especies de cucurbitáceas y en otras regiones del país.

Además, es necesario desarrollar estudios epidemiológicos de GRSV en sandía, ya que evaluar los riesgos de una nueva enfermedad es un punto importante para el futuro en el cultivo de cucurbitáceas.

\section{AGRADECIMIENTOS}

Este trabajo fue realizado con fondos provenientes de proyectos INTA y CONICET, quienes además pagaron el sueldo para los investigadores y el otorgamiento de becas para algunos de los autores del trabajo. Se agradece también especialmente a los productores agrícolas que facilitaron los muestreos en los lotes de producción y al personal de las EEA Santiago del Estero - INTA que colaboraron con la recolección de las muestras y recorridos por los cultivos.

\section{BIBLIOGRAFÍA}

Bag, S., Mitter, N., Eid, S. y Pappu, H. R. (2012). Complementation between Two Tospoviruses Facilitates the Systemic Movement of a Plant Virus Silencing Suppressor in an Otherwise Restrictive Host. PLOS ONE, 7 (10), e44803. DOI: https://doi. org/10.1371/journal.pone.0044803

Bezerra, I. C., Resende, R. O., Pozzer, L., Nagata, T., Kormelink, R. y De Avila, A. C. (1999). Increase of tospoviral diversity in Brazil with the identification of two new Tospovirus species, one from chrysanthemum and one from zucchini. Phytopathology,89 (9), 823830. DOI: https://doi.org/10.1094/phyto.1999.89.9.823

Basic Local Alignment Search Tool. [Software]. Maryland, EE.UU.: U.S. National Libarary of Medicine. National Center for Biotechnology Information. https://blast. ncbi.nlm.nih.gov/Blast.cgi.

Boari, A. J., Maciel-Zambolim, E., Lau, D. D., Lima, G. S. A., Kitajima, E. W., Brommonschenkel, S. S. H. y Zerbini, F. M. (2002). Detection and partial characterization of an isolate of Groundnut ringspot virus in Solanum sessiliflorum. Fitopatologia Brasileira, 27 (3), 249-253. DOI: https://doi.org/10.1590/S0100- 


\section{2}

Ciuffo, M., Kurowski, C., Vivoda, E., Copes, B., Masenga, V., Falk, B. W. y Turina, M. (2009). A new Tospovirus sp. in cucurbit crops in Mexico. Plant Disease, 93 (3), 467-474. DOI: https://doi.org/10.1094/PDIS-93-5-0467

Clark, M. F. y Adams, A. N. (1977). Characteristics of the microplate method of enzyme-linked immunosorbent assay for the detection of plant viruses. Journal of General Virology,34 (3), 475-483. DOI:https://doi. org/10.1099/0022-1317-34-3-475

de Avila, A. C., de Haan, P., Kormelink, R., Resende, R. O., Goldbach, R. W. y Peters, D. (1993). Classification of tospoviruses based on phylogeny of nucleoprotein gene sequences. Journal of General Virology, 74 (2), 153-159. DOI: https://doi.org/10.1099/0022-1317-742-153

de Breuil, S., Abad, J. A., Nome, C. F., Giolitti, F. J., Lambertini, P. L. y Lenardon, S. (2007). Groundnut ringspot virus: an emerging tospovirus inducing disease in peanut crops. Journal of Phytopathology, 155 (2), 251-254. DOI: https://doi.org/10.1111/j.14390434.2007.01221.x

Dewey, R. A., Semorile, L.C. y Grau, O. (1996). Detection of Tospovirus species by RT-PCR of the N-gene and restriction enzyme digestions of the products. Journal of Virological Methods, 56, 19-26. DOI: https://doi. org/10.1016/0166-0934(95)01896-4

Gambino, G., Perrone, I. y Gribaudo, I. (2008). A rapid and effective method for RNA extraction from different tissues of grapevine and other woody plants. Phytochemical Analysis, 19 (6), 520-525. DOI: https:// doi.org/10.1002/pca.1078

German, T., Ullman, D. y Moyer, J. (1992). Tospoviruses: Diagnosis, Molecular Biology, Phylogeny, and Vector Relationships. Annual Review of Phytopathology, 30, 315-348. DOI: https://doi.org/10.1146/annurev. py.30.090192.001531

Goldbach, R. y Kuo, G. (1996). Introduction. Acta Horticulturae, 431, 21-26. DOI: https://doi. org/10.17660/ActaHortic. 1996.431.1

Gracia, O. y Feldman, J. M. (1986). Virus identificados en cultivos de cucurbitáceas. IDIA 445-448, 1-6.

Holkar, S. K., Mandal, B., Reddy, M. K. y Jain, R. K. (2019). Watermelon bud necrosis orthotospovirus. An emerging constraint in the Indian subcontinent: An overview. Crop Protection, 117, 52-62. D069+l: https:// doi.org/10.1016/i.cropro.2018.11.005

Jain, R. K., Pappu, H. R., Pappu, S. S., Reddy, M. K. y Vani, A. (1998). Watermelon bud necrosis tospovirus is a distinct virus species belonging to serogroup IV. Archives of Virology, 143, 1637-1644. DOI: https://doi. org/10.1007/s007050050405

Jones, D. R. (2005). Plant viruses transmitted by thrips.
European Journal of Plant Pathology, 113, 119-157. DOI: https://doi.org/10.1007/s10658-005-2334-1

Kato, K., Handa, K. y Kameya-Iwaki, M. (2000). Melon yellow spot virus: a distinct species of the genus Tospovirus isolated from melon. Phytopathology, 90 (4), 422-426. DOI: https://doi.org/10.1094/ PHYTO.2000.90.4.422

Kumar, S., Stecher, G. y Tamura, K. MEGA7: molecular evolutionary genetics analysis version 7.0 for bigger datasets. (version 7.0) [Software]. Recuperado de: https://www.megasoftware.net/

Leão, E., Spadotti, D., Rocha, K., Pantoja, K., Rezende, J., Pavan, M., y Krause Sakate, R. (2015). Citrullus lanatus is a new natural host of Groundnut ringspot virus in Brazil. Journal of Phytopathology, 163 (11-12), 1014-1018. DOI: https://doi.org/10.1111/jph.12327

Lecoq, H. y Katis, N. (2014). Control of cucurbit viruses. Advances in Virus Research, 90, 255-296. DOI: https:/l doi.org/10.1016/B978-0-12-801246-8.00005-6

Loebenstein, G. y Lecoq, H. (Eds.) (2012). Viruses and virus diseases of vegetables in the Mediterranean basin. (Vol. 84). Estados Unidos: Academic Press.

Maes, P., Adkins, S., Alkhovsky, S. V., Avši -Županc, T., Ballinger, M. J., Bente, D. A., ...y Kuhn, J. H. (2019). Taxonomy of the order Bunyavirales: second update 2018. Archives of Virology, 164, 927-941. DOI: https:// doi.org/10.1007/s00705-018-04127-3

Michelotto, M., Carrega, W., Lamana, L., de Souza, T., de Godoy, I., dos Reis, L., ... y Carvalho, R. (2019). Losses caused by Groundnut ringspot tospovirus in peanut crop in the State of São Paulo. Semina. Ciências Agrárias, 40 (6), 3429-3442. DOI: http:/l dx.doi.org/10.5433/1679-0359.2019v40n6Supl3p3429

Milne,J. R., Khumlekhasing, M. y Walter, G. H. (1996). Understanding host plant relationships of polyphagous flower thrips, a case study of Frankliniella schultzei (Trybom). En S. Goodwin and P. Gillespie (Eds.), Proceedings of the 1995 Australia and New Zealand Thrips Workshop: Methods, Ecology and Management ) (pp. 8-14). Gosford, Australia: New South Wales Agriculture.

Mumford, R. A., Barker, I. y Wood, K. R. (1996). The biology of the tospoviruses. Annals of Applied Biology, 128, 159-183. DOI: https://doi. org/10.1111/j.1744-7348.1996.tb07097.x

Nagata, T., Almeida, A. C. L., Resende, R. D. O. y De Ávila, A. C. (2004). The competence of four thrips species to transmit and replicate four tospoviruses. Plant Pathology, 53 (2), 136-140. DOI: https://doi. org/10.1111/j.0032-0862.2004.00984.x

Nome, S. F., March, G. J. y Giorda, L. M. (1974). Disminución de la productividad de plantas de zapallito de tronco infectadas con el virus del mosaico de la sandía, raza 2 (Watermelon Mosaic Virus-2). IDIA 
321-324, 26-31

Olaya, C., Adhikari, B., Raikhy, G., Cheng, J. y Pappu, H. R. (2019). Identification and localization of Tospovirus genus-wide conserved residues in 3D models of the nucleocapsid and the silencing suppressor proteins. Virology Journal, 16, 7.DOI: https://doi.org/10.1186/ s12985-018-1106-4

Oliver, J. E. y Whitfield, A. E. (2016). The Genus Tospovirus : Emerging Bunyaviruses that Threaten Food Security. Annual Review of Virology, 3, 101-124. DOI: https:// doi.org/10.1146/annurev-virology-100114-055036

Pappu, H. R., Jones, R. A. C. y Jain, R. K. (2009). Global status of tospovirus epidemics in diverse cropping systems: successes achieved and challenges ahead. Virus Research, 141 (2), 219-236. DOI: https://doi. org/10.1016/j.virusres.2009.01.009

Perotto, M. C., Celli, M. G., Pozzi, E. A., Luciani, C. E. y Conci, V. C. (2016). Occurrence and characterization of a severe isolate of Watermelon mosaic virus from Argentina. European Journal of Plant Pathology, 146, 213-218. DOI: https://doi.org/10.1007/s10658-0160904-z

Perotto, M. C., Pozzi, E. A., Celli, M. G., Luciani, C. E., Mitidieri, M. S. y Conci, V. C. (2018). Identification and characterization of a new potyvirus infecting cucurbits. Archives of Virology, 163, 719-724. DOI: https://doi. org/10.1007/s00705-017-3660-2

Plyusnin, A., Beaty, B. J., Elliott, R. M., Goldbach, R., Kormelink, R., Lundkvist, A.,... y Tesh, R .B. (2011). Bunyaviridae. In A. M. Q. King, M. J. Adams, E. B. Carstens, E. J. Lefkowitz (Eds.), Ninth Report of the International Committee on Taxonomy of Viruses. Recuperado de: https://talk.ictvonline.org/ictv-reports/ ictv 9th report/negative-sense-rna-viruses-2011/w/ negrna_viruses/205/bunyaviridae

Pozzi, E. A., Luciani, C. E., Celli, M. G., Conci, V. C., y Perotto, M. C. (2019). First Report of Zucchini Lethal Chlorosis Virus in Argentina Infecting Squash Crops. Plant Disease, 104 (2), 609. DOI: https://doi. org/10.1094/PDIS-05-19-1064-PDN

Pretty, J. (2007). Agricultural sustainability: concepts, principles and evidence. Philosophical Transactions of the Royal Society B: Biological Sciences, 363(1491), 447465. DOI: https://doi.org/10.1098/rstb.2007.2163

Rao, X., Liu, Y., Wu, Z., y Li, Y. (2011). First report of natural infection of watermelon by Watermelon silver mottle virus in China. New Disease Reports, 24, 2044-0588. DOI: https://doi.org/10.5197 /j.2044-0588.2011.024.012

Riley, D. G., Joseph, S. V., Srinivasan, R. y Diffie, S. (2011). Thrips Vectors of Tospoviruses. Journal of Integrated Pest Management, 2, I1-|10. DOI: https:/l doi.org/10.1603/IPM10020
Rotenberg, D., Jacobson, A. L., Schneweis, D. J. y Whitfield, A. E. (2015). Thrips transmission of tospoviruses. Current Opinion in Virology, 15, 80-89. DOI: https://doi.org/10.1016/j.coviro.2015.08.003

Sistema Nacional Argentino de Vigilancia y Monitoreo de plagas (SINAVIMO) (2019a). Frankliniella schultzei. Recuperado de https://www.sinavimo.gov.ar/plagal frankliniella-schultzei

Sistema Nacional Argentino de Vigilancia y Monitoreo de plagas (SINAVIMO) (2019 b). Frankliniella occidentalis. Recuperado de https://www.sinavimo.gov.ar/plagal frankliniella-occidentalis

Sistema Nacional Argentino de Vigilancia y Monitoreo de plagas (SINAVIMO) (2019 c). Groundnut ring spot virus (GRSV). Recuperado de https://www.sinavimo. gov.ar/plaga/groundnut-ring-spot-virus

Spadotti, D., Leão, E., Rocha, K., Pavan, M. y KrauseSakate, R. (2014). First report of Groundnut ringspot virus in cucumber fruits in Brazil. New Disease Reports,29, 25. DOI: http://dx.doi.org/10.5197 /j.2044-0588.2014.029.025

Tsompana, M., Abad, J., Purugganan, M., y Moyer, J. W. (2005). The molecular population genetics of the Tomato spotted wilt virus (TSWV) genome. Molecular Ecology, 14, 53-66. DOI: https://doi.org/10.1111/ j.1365-294X.2004.02392.x

Turina, M., Tavella, L. y Ciuffo, M. (2012). Tospoviruses in the Mediterranean area. Advances in Virus Research, 84, 403-437. DOI: https://doi.org/10.1016/B978-0-12394314-9.00012-9

Webster, C. G., Frantz, G., Reitz, S. R., Funderburk, J. E., Mellinger, H. C., McAvoy, E., .... y Adkins, S. (2015). Emergence of Groundnut ringspot virus and Tomato chlorotic spot virus in vegetables in Florida and the southeastern United States. Phytopathology, 105 (3), 388-398. DOI: https://doi.org/10.1094/PHYTO-06-140172-R

Wijkamp, I., Almarza, N., Goldbach, R. y Peters, D. (1995). Distinct levels of specificity in thrips transmission of tospoviruses. Phytopathology,85 (10), 1069-1074. DOI: https://doi.org/10.1094/Phyto-85-1069

Yeh, S. D., Lin, Y. C., Cheng, Y. H., Jih, C. L., Chen, M. J. y Chen, C. C. (1992). Identification of tomato spotted wilt-like virus on watermelon in Taiwan. Plant Disease, 76, 835-840. DOI: https://doi.org/10.1094/PD-76-0835

Yin, Y., Zheng, K., Dong, J., Fang, Q., Wu, S., Wang, L. y Zhang, Z. (2014). Identification of a new tospovirus causing necrotic ringspot on tomato in China. Virology Journal, 11, 213. DOI: https://doi.org/10.1186/s12985014-0213-0 